\title{
A UNIFIED APPROACH TO STOCHASTIC EVOLUTION EQUATIONS USING THE SKOROKHOD INTEGRAL
}

\author{
S. V. LOTOTSKY AND B. L. ROZOVSKII
}

\begin{abstract}
We study stochastic evolution equations driven by Gaussian noise. The key features of the model are that the operators in the deterministic and stochastic parts can have the same order and the noise can be time-only, space-only, or spacetime. Even the simplest equations of this kind do not have a square-integrable solution and must be solved in special weighted spaces. We demonstrate that the Cameron-Martin version of the Wiener chaos decomposition leads to natural weights and a natural replacement of the square integrability condition.
\end{abstract}

October 29, 2018

\section{INTRODUCTION}

Let $W$ be a standard Brownian motion, $u_{0} \in L_{2}(\mathbb{R})$, a deterministic function, and $\sigma$, a real number. It is well known that the Itô equation

$$
u(t, x)=u_{0}(x)+\int_{0}^{t} u_{x x}(s, x) d s+\int_{0}^{t} \sigma u(s, x) d W(s)
$$

has a solution $u(t, x)$ and

$$
\int_{\mathbb{R}} \mathbb{E} u^{2}(t, x) d x<\infty
$$

for every $t>0$ and all $\sigma \in \mathbb{R}$; see, for example, [10] or simply consider the Fourier transform of the equation. On the other hand, the solution of equation

$$
u(t, x)=u_{0}(x)+\int_{0}^{t} u_{x x}(s, x) d s+\int_{0}^{t} \sigma u_{x}(s, x) d W(s)
$$

satisfies (1.2) for an arbitrary $u_{0} \in L_{2}(\mathbb{R})$ if and only if $|\sigma| \leq \sqrt{2}$, while equation

$$
u(t, x)=u_{0}(x)+\int_{0}^{t} u_{x x}(s, x) d s+\int_{0}^{t} \sigma u_{x x}(s, x) d W(s)
$$

hardly ever has a solution satisfying (1.2). Still, because of the Itô integral, all three equations share a common feature, namely, that the mean evolution is described by the heat equation. One of the objectives of this work is to develop a unified approach

2000 Mathematics Subject Classification. Primary 60H15; Secondary 35R60, 60H40.

Key words and phrases. Generalized Random Elements, Malliavin Calculus, Wick Product, Wiener Chaos, Weighted Spaces.

S. V. Lototsky acknowledges support from the NSF CAREER award DMS-0237724.

B. L. Rozovskii acknowledges support from NSF Grant DMS 0604863, ARO Grant W911NF-071-0044, and ONR Grant N00014-07-1-0044. 
that would cover all three equations above without any special restrictions on the initial condition or the amplitude of noise.

The situation becomes even more complicated when the noise has a spacial component. As an illustration, take a standard random variable $\xi$ and consider the equation

$$
u(t)=1+\int_{0}^{t} u(s) d s+\int_{0}^{t} u(s) \xi d s .
$$

The solution is clearly $u(t)=e^{t} e^{\xi t}$. The unperturbed solution is $e^{t}$, so that $\mathbb{E} u(t) \neq e^{t}$. It turns out that, to preserve the mean dynamics, the usual product $u \xi$ must be replaced by the Wick product $u \diamond \xi$. In particular, the solution of

$$
u(t)=1+\int_{0}^{t} u(s) d s+\int_{0}^{t} u(s) \diamond \xi d s
$$

is $u(t)=e^{t} e^{\xi t-\left(t^{2} / 2\right)}$ and satisfies $\mathbb{E} u(t)=e^{t}$. For partial differential equations of this type, such as

$$
u(t, x)=u_{0}(x)+\int_{0}^{t} u_{x x}(s, x) d s+\int_{0}^{t} u_{x}(s, x) \diamond \xi d s,
$$

inequality (1.2), without additional assumptions on $u_{0}$, holds only for sufficiently small $t$. Still, the same framework that unifies equations (1.1), (1.3), (1.4), also covers (1.5), (1.6), and a wide class of other equations with space and space-time Gaussian noise.

There are three main components in the general construction presented in this paper:

(1) Use of the Skorokhod integral $\boldsymbol{\delta}$ for all types of random perturbation.

(2) Separation of the time as the variable in the equation from the possible time evolution of the noise.

(3) Replacement of the space $L_{2}(\Omega ; X)$ of square-integrable $X$-valued processes with a space $(\mathcal{L})_{Q, r}(X)$ of generalized (and not necessarily adapted) random elements as the space for both the input data and the solution.

Section 2 presents the necessary definitions and constructions related to the Skorokhod integral with respect to an abstract Gaussian white noise and Section 3 introduces the spaces $(\mathcal{L})_{Q, r}$. Section 4 presents the unified treatment of stochastic evolution equations. While the main results, Theorem 4.7 cannot be fully understood without all the preliminaries, there is a certain analogy with the corresponding result for the deterministic equations. Namely, let $\left(V, H, V^{\prime}\right)$ be a normal triple of Hilbert spaces (defined precisely in Section 4) and let $\mathbf{A}(t), t \in[0, T]$ be a family of bounded linear operators from $V$ to $V^{\prime}$. It is known from deterministic analysis, that if the family of operators $\mathbf{A}(t)$ is uniformly elliptic, then the initial value problem for the equation $u(t)=u_{0}+\int_{0}^{t}(\mathbf{A}(s) u(s)+f(s)) d s$ is well-posed in the normal triple $\left(V, H, V^{\prime}\right)$.

Now consider the initial value problem for the stochastic equation

$$
u(t)=u_{0}+\int_{0}^{t}(\mathbf{A}(s) u(s)+f(s)) d s+\int_{0}^{t} \boldsymbol{\delta}(\mathbf{M}(s) u(s)) d s .
$$


The statement of Theorem 4.7 is essentially as follows: if the family of operators $\mathbf{A}(t)$ is uniformly elliptic and $\mathbf{M}(t)$ is a family of continuous operators from $V$ to $V^{\prime}$, then the initial value problem (1.7) is well posed in the normal triple $\left((\mathcal{L})_{Q, r}(V),(\mathcal{L})_{Q, r}(H),(\mathcal{L})_{Q, r}\left(V^{\prime}\right)\right)$. The reader should not miss Example 4.4 illustrating how many familiar equations are particular cases of (1.7).

\section{Skorokhod Integral With Respect to Gaussian White Noise}

Let $\mathbb{F}=(\Omega, \mathcal{F}, \mathbb{P})$ be a complete probability space, and $\mathcal{U}$, a real separable Hilbert space with inner product $(\cdot, \cdot)_{\mathcal{U}}$. On $\mathbb{F}$, consider a zero-mean Gaussian family

$$
\dot{W}=\{\dot{W}(h), h \in \mathcal{U}\}
$$

so that

$$
\mathbb{E}\left(\dot{W}\left(h_{1}\right) \dot{W}\left(h_{2}\right)\right)=\left(h_{1}, h_{2}\right) \mathcal{u}
$$

It suffices, for our purposes, to assume that $\mathcal{F}$ is the $\sigma$-algebra generated by $\dot{W}(h), h \in \mathcal{U}$. Given a real separable Hilbert space $X$, we denote by $L_{2}(\mathbb{F} ; X)$ the Hilbert space of square-integrable $\mathcal{F}$-measurable $X$-valued random elements $f$. In particular,

$$
(f, g)_{L_{2}(\mathbb{F} ; X)}^{2}:=\mathbb{E}(f, g)_{X}^{2} .
$$

When $X=\mathbb{R}$, we write $L_{2}(\mathbb{F})$ instead of $L_{2}(\mathbb{F} ; \mathbb{R})$.

Definition 2.1. A formal series

$$
\dot{W}=\sum_{k \geq 1} \dot{W}\left(\mathfrak{u}_{k}\right) \mathfrak{u}_{k}
$$

where $\left\{\mathfrak{u}_{k}, k \geq 1\right\}$ is a complete orthonormal basis in $\mathcal{U}$, is called Gaussian white noise on $\mathcal{U}$.

Example 2.2. If $\mathcal{U}=L_{2}((0, T))$, then $\dot{W}(h)=\int_{0}^{T} h(s) d w(s)$, where $w(t)=\dot{W}\left(\chi_{t}\right)$ is the standard Brownian motion; $\chi_{t}$ is the characteristic function of the interval $[0, t]$.

Given an orthonormal basis $\mathfrak{U}=\left\{\mathfrak{u}_{k}, k \geq 1\right\}$ in $\mathcal{U}$, define a collection $\left\{\xi_{k}, k \geq 1\right\}$ of independent standard Gaussian random variables by $\xi_{k}=\dot{W}\left(\mathfrak{u}_{k}\right)$. Let $\mathcal{J}$ be the collection of multi-indices $\alpha$ with $\alpha=\left(\alpha_{1}, \alpha_{2}, \ldots\right)$ so that each $\alpha_{k}$ is a non-negative integer and $|\alpha|:=\sum_{k \geq 1} \alpha_{k}<\infty$. For $\alpha, \beta \in \mathcal{J}$, we define

$$
\alpha+\beta=\left(\alpha_{1}+\beta_{1}, \alpha_{2}+\beta_{2}, \ldots\right), \quad \alpha !=\prod_{k \geq 1} \alpha_{k} !
$$

By (0) we denote the multi-index with all zeroes. By $\varepsilon_{i}$ we denote the multi-index $\alpha$ with $\alpha_{i}=1$ and $\alpha_{j}=0$ for $j \neq i$. With this notation, $n \varepsilon_{i}$ is the multi-index $\alpha$ with $\alpha_{i}=n$ and $\alpha_{j}=0$ for $j \neq i$.

Define the collection of random variables $\Xi=\left\{\xi_{\alpha}, \alpha \in \mathcal{J}\right\}$ as follows:

$$
\xi_{\alpha}=\prod_{k}\left(\frac{H_{\alpha_{k}}\left(\xi_{k}\right)}{\sqrt{\alpha_{k} !}}\right),
$$


where

$$
H_{n}(x)=(-1)^{n} e^{x^{2} / 2} \frac{d^{n}}{d x^{n}} e^{-x^{2} / 2}
$$

is Hermite polynomial of order $n$.

Theorem 2.3 (Cameron and Martin [1]). The collection $\Xi=\left\{\xi_{\alpha}, \alpha \in \mathcal{J}\right\}$ is an orthonormal basis in $L_{2}(\mathbb{F} ; X)$ : if $\eta \in L_{2}(\mathbb{F} ; X)$ and $\eta_{\alpha}=\mathbb{E}\left(\eta \xi_{\alpha}\right)$, then $\eta=\sum_{\alpha \in \mathcal{J}} \eta_{\alpha} \xi_{\alpha}$ and $\mathbb{E}|\eta|^{2}=\sum_{\alpha \in \mathcal{J}} \eta_{\alpha}^{2}$

Denote by $\mathbf{D}$ the Malliavin derivative on $L_{2}(\mathbb{F})$ (see e.g. [9]). In particular, if $F$ : $\mathbb{R}^{N} \rightarrow \mathbb{R}$ is a smooth function and $h_{i} \in \mathcal{U}, i=1, \ldots N$, then

$$
\mathbf{D} F\left(\dot{W}\left(h_{1}\right), \ldots \dot{W}\left(h_{N}\right)\right)=\sum_{i=1}^{N} \frac{\partial F}{\partial x_{i}}\left(\dot{W}\left(h_{1}\right), \ldots, \dot{W}\left(h_{N}\right)\right) h_{i} \in L_{2}(\mathbb{F} ; \mathcal{U}) .
$$

It is known [9] that the domain $\mathbb{D}^{1,2}(\mathbb{F})$ of the operator $\mathbf{D}$ is a dense linear subspace of $L_{2}(\mathbb{F})$.

The adjoint of the Malliavin derivative on $L_{2}(\mathbb{F})$ is the Skorokhod integral and is traditionally denoted by $\boldsymbol{\delta}[9]$. The operator $\boldsymbol{\delta}$ extends to a subspace $\mathbb{D}^{1,2}(\mathbb{F} ; X \otimes \mathcal{U})$ of $L_{2}(\mathbb{F} ; X \otimes \mathcal{U})$ for a Hilbert space $X$ : given $f \in \mathbb{D}^{1,2}(\mathbb{F} ; X \otimes \mathcal{U}), \boldsymbol{\delta}(f)$ is the unique element of $L_{2}(\mathbb{F} ; X)$ with the property

$$
\mathbb{E}(\varphi \boldsymbol{\delta}(f))=\mathbb{E}(f, \mathbf{D} \varphi)_{\mathcal{U}}
$$

for all $\varphi \in \mathbb{D}^{1,2}(\mathbb{F})$.

We will now derive the expressions for the operators $\mathbf{D}$ and $\boldsymbol{\delta}$ in the basis $\Xi$. To begin, let us compute $\mathbf{D}\left(\xi_{\alpha}\right)$.

Proposition 2.4. For each $\alpha \in \mathcal{J}$,

$$
\mathbf{D}\left(\xi_{\alpha}\right)=\sum_{k \geq 1} \sqrt{\alpha_{k}} \xi_{\alpha-\varepsilon_{k}} \mathfrak{u}_{k}
$$

Proof. The result follows by direct computation using the property (2.4) of the Malliavin derivative and the relation $H_{n}^{\prime}(x)=n H_{n-1}(x)$ for the Hermite polynomials (cf. [9, Chapter 1]).

Remark 2.5. The set $\mathcal{J}$ is not closed under substraction and the expression $\alpha-\varepsilon_{k}$ is undefined if $\alpha_{k}=0$. Everywhere in this paper when undefined expressions of this type appear, we use the following convention: if $\alpha_{k}=0$, then $\sqrt{\alpha_{k}} \xi_{\alpha-\varepsilon_{k}}=0$.

Proposition 2.6. For $\xi_{\alpha} \in \Xi, h \in X$, and $\mathfrak{u}_{k} \in \mathfrak{U}$,

$$
\boldsymbol{\delta}\left(\xi_{\alpha} h \otimes \mathfrak{u}_{k}\right)=h \sqrt{\alpha_{k}+1} \xi_{\alpha+\varepsilon_{k}} .
$$

Proof. It is enough to verify (2.5) with $f=h \otimes \mathfrak{u}_{k} \xi_{\alpha}$ and $\varphi=\xi_{\beta}$, where $h \in X$. By $(2.6)$,

$$
\mathbb{E}(f, \mathbf{D} \varphi)_{\mathcal{U}}=\sqrt{\beta_{k}} h \mathbb{E}\left(\xi_{\alpha} \xi_{\beta-\varepsilon_{k}}\right)= \begin{cases}\sqrt{\alpha_{k}+1} h, & \text { if } \alpha=\beta-\varepsilon_{k} \\ 0, & \text { if } \alpha \neq \beta-\varepsilon_{k}\end{cases}
$$


In other words,

$$
\mathbb{E}\left(\xi_{\alpha} h \otimes \mathfrak{u}_{k}, \mathbf{D} \xi_{\beta}\right) \mathfrak{U}=h \mathbb{E}\left(\sqrt{\alpha_{k}+1} \xi_{\alpha+\varepsilon_{k}} \xi_{\beta}\right)
$$

for all $\beta \in \mathcal{J}$.

Remark 2.7. The operator $\boldsymbol{\delta} \mathbf{D}$ is linear and unbounded on $L_{2}(\mathbb{F})$; it follows from Propositions 2.4 and 2.6 that the random variables $\xi_{\alpha}$ are eigenfunctions of this operator:

$$
\boldsymbol{\delta}\left(\mathbf{D}\left(\xi_{\alpha}\right)\right)=|\alpha| \xi_{\alpha}
$$

To give an alternative characterization of the operator $\boldsymbol{\delta}$, we define a new operation on the elements of $\Xi$.

Definition 2.8. For $\xi_{\alpha}, \xi_{\beta}$ from $\Xi$, define the Wick product

$$
\xi_{\alpha} \diamond \xi_{\beta}:=\sqrt{\left(\frac{(\alpha+\beta) !}{\alpha ! \beta !}\right)} \xi_{\alpha+\beta} .
$$

In particular, taking in (2.8) $\alpha=k \varepsilon_{i}$ and $\beta=n \varepsilon_{i}$, and using (2.2), we get

$$
H_{k}\left(\xi_{i}\right) \diamond H_{n}\left(\xi_{i}\right)=H_{k+n}\left(\xi_{i}\right) \text {. }
$$

An immediate consequence of Proposition 2.6 and Definition 2.8 is the following identity:

$$
\boldsymbol{\delta}\left(\xi_{\alpha} h \otimes \mathfrak{u}_{k}\right)=h \xi_{\alpha} \diamond \xi_{k}, h \in X .
$$

More generally, we define the operation $\diamond$ on formal series:

$$
\left(\sum_{\alpha \in \mathcal{J}} f_{\alpha} \xi_{\alpha}\right) \diamond\left(\sum_{\alpha \in \mathcal{J}} g_{\alpha} \xi_{\alpha}\right)=\sum_{\alpha \in \mathcal{J}}\left(\sum_{\beta, \gamma \in \mathcal{J}: \beta+\gamma=\alpha} \sqrt{\frac{\alpha !}{\beta ! \gamma !}} f_{\beta} g_{\gamma}\right) \xi_{\alpha}
$$

for $f_{\alpha} \in X, g_{\alpha} \in \mathbb{R}$.

Theorem 2.9. If $f=\sum_{k \geq 1} f_{k} \otimes \mathfrak{u}_{k}, f_{k}=\sum_{\alpha \in \mathcal{J}} f_{k, \alpha} \xi_{\alpha} \in \mathcal{R} L_{2}(\mathbb{F} ; X)$, and $f$ is in the domain of $\boldsymbol{\delta}$, then

$$
\boldsymbol{\delta}(f)=\sum_{k \geq 1} f_{k} \diamond \xi_{k}
$$

and

$$
(\boldsymbol{\delta}(f))_{\alpha}=\sum_{k \geq 1} \sqrt{\alpha_{k}} f_{k, \alpha-\varepsilon_{k}}
$$

Proof. By linearity and (2.11),

$$
\boldsymbol{\delta}(f)=\sum_{k \geq 1} \sum_{\alpha \in \mathcal{J}} \boldsymbol{\delta}\left(\xi_{\alpha} f_{k, \alpha} \otimes \mathfrak{u}_{k}\right)=\sum_{k \geq 1} \sum_{\alpha \in \mathcal{J}} f_{k, \alpha} \xi_{\alpha} \diamond \xi_{k}=\sum_{k \geq 1} f_{k} \diamond \xi_{k},
$$

which is (2.12). On the other hand, by (2.7),

$$
\boldsymbol{\delta}(f)=\sum_{k \geq 1} \sum_{\alpha \in \mathcal{J}} f_{k, \alpha} \sqrt{\alpha_{k}+1} \xi_{\alpha+\varepsilon_{k}}=\sum_{k \geq 1} \sum_{\alpha \in \mathcal{J}} f_{k, \alpha-\varepsilon_{k}} \sqrt{\alpha_{k}} \xi_{\alpha},
$$

and (2.13) follows. 
To proceed, we need a description of a multi-index $\alpha$ with $|\alpha|=n>0$ using its characteristic set $K_{\alpha}$, that is, an ordered $n$-tuple $K_{\alpha}=\left\{k_{1}, \ldots, k_{n}\right\}$, where $k_{1} \leq k_{2} \leq$ $\ldots \leq k_{n}$ characterize the locations and the values of the non-zero elements of $\alpha$. More precisely, $k_{1}$ is the index of the first non-zero element of $\alpha$, followed by $\max \left(0, \alpha_{k_{1}}-1\right)$ of entries with the same value. The next entry after that is the index of the second non-zero element of $\alpha$, followed by $\max \left(0, \alpha_{k_{2}}-1\right)$ of entries with the same value, and so on. For example, if $n=7$ and $\alpha=(1,0,2,0,0,1,0,3,0, \ldots)$, then the non-zero elements of $\alpha$ are $\alpha_{1}=1, \alpha_{3}=2, \alpha_{6}=1, \alpha_{8}=3$. As a result, $K_{\alpha}=\{1,3,3,6,8,8,8\}$, that is, $k_{1}=1, k_{2}=k_{3}=3, k_{4}=6, k_{5}=k_{6}=k_{7}=8$. Note also that, for every sequence $\left(b_{k}, k \geq 1\right)$ of positive numbers,

$$
\prod_{k \geq 1} b_{k}^{\alpha_{k}}=b_{i_{1}} \cdot b_{i_{2}} \cdot \ldots \cdot b_{i_{n}}=\prod_{i \in K_{\alpha}} b_{i}
$$

which can serve as an equivalent definition of $K_{\alpha}$.

Using the notion of the characteristic set, we now state the following analog of the wellknown result of Itô [3] connecting multiple Wiener integrals and Hermite polynomials.

Proposition 2.10. Let $\alpha \in \mathcal{J}$ be a multi-index with $|\alpha|=n \geq 1$ and characteristic set $K_{\alpha}=\left\{k_{1}, \ldots, k_{n}\right\}$. Then

$$
\xi_{\alpha}=\frac{\xi_{k_{1}} \diamond \xi_{k_{2}} \diamond \cdots \diamond \xi_{k_{n}}}{\sqrt{\alpha !}}
$$

Proof. This follows from (2.2) and (2.10), because by (2.10), for every $i$ and $k$,

$$
H_{k}\left(\xi_{i}\right)=\underbrace{\xi_{i} \diamond \cdots \diamond \xi_{i}}_{k \text { times }} \text {. }
$$

By induction, we define the operator $\boldsymbol{\delta}^{\hat{\otimes} n}, n>1$ on the space $L_{2}\left(\mathbb{F} ; X \otimes \mathcal{U}^{\hat{\otimes} n}\right)$, where $\mathcal{U}^{\hat{\otimes} n}$ is the symmetric tensor power of $\mathcal{U}$. Then relation (2.11) leads to an alternative form of (2.15):

$$
\xi_{\alpha}=\frac{1}{|\alpha| ! \sqrt{\alpha !}} \boldsymbol{\delta}^{\hat{\otimes}|\alpha|}\left(E_{\alpha}\right)
$$

where

$$
E_{\alpha}=\sum_{\sigma \in \mathcal{P}} \mathfrak{u}_{i_{\sigma(1)}} \otimes \cdots \otimes \mathfrak{u}_{i_{\sigma(n)}}
$$

with summation in (2.17) taken over all permutations $\mathcal{P}$ of the set $\{1, \ldots, n\}$. This leads to the following generalization of the multiple Wiener integral expansion for the elements of $L_{2}(\mathbb{F} ; X)$.

Theorem 2.11. If $\eta \in L_{2}(\mathbb{F} ; X)$, then there is a unique collection of the deterministic elements $\eta_{k}, k \geq 0$, with $\eta_{0}=\mathbb{E} \eta \in X$ and $\eta_{k} \in X \otimes \mathcal{U}^{\hat{\otimes} k}, k \geq 1$, with the properties

$$
\eta=\eta_{0}+\sum_{k \geq 1} \frac{1}{k !} \boldsymbol{\delta}^{\hat{\otimes} k}\left(\eta_{k}\right), \quad \mathbb{E}\|\eta\|_{X}^{2}=\left\|\eta_{0}\right\|_{X}^{2}+\sum_{k \geq 1} \frac{1}{k !}\|\| \eta_{k}\left\|_{\mathcal{U}^{\hat{\otimes} n}}\right\|_{X}^{2}
$$


Proof. Using (2.16) and Theorem 2.3,

$$
\eta=\eta_{0}+\sum_{k=1}^{\infty} \frac{1}{k !} \sum_{|\alpha|=k} \eta_{\alpha} \boldsymbol{\delta}^{\hat{\otimes} k}\left(\frac{1}{\sqrt{\alpha !}} E_{\alpha}\right),
$$

and we get the first equality in (2.18) with

$$
\eta_{k}=\sum_{|\alpha|=k} \boldsymbol{\delta}^{\hat{\otimes} k}\left(\frac{1}{\sqrt{\alpha !}} \eta_{\alpha} \otimes E_{\alpha}\right) .
$$

The second equality in (2.18) now follows from

$$
\mathbb{E}\|\eta\|_{X}^{2}=\left\|\eta_{0}\right\|_{X}^{2}+\sum_{k \geq 1} \sum_{|\alpha|=k}\left\|\eta_{\alpha}\right\|_{X}^{2},
$$

because, by direct computation,

$$
\left\|E_{\alpha}\right\|_{\mathcal{U}^{\otimes} n}^{2}=k ! \alpha !
$$

3. The Spaces $(\mathcal{L})_{Q, r}$

Definition 3.1. A sequence

$$
Q=\left\{q_{k}, k \geq 1\right\}
$$

is called $a$ weight sequence if $q_{k} \geq 1$ for all $k \geq 1$.

For $\alpha \in \mathcal{J}$ and a real number $r$ we write

$$
q^{r \alpha}=\prod_{k \geq 1} q_{k}^{r \alpha_{k}}
$$

Given a weight sequence $Q$, denote by $\Lambda_{Q}$ the following self-adjoint operator on $\mathcal{U}$ :

$$
\Lambda_{Q} \mathfrak{u}_{k}=q_{k} \mathfrak{u}_{k}, \quad k \geq 1
$$

Then, for every $r \in \mathbb{R}$, the operator $\Lambda_{Q}^{r}$ is defined. The domain of every $\Lambda_{Q}^{r}$ contains finite linear combinations of $\mathfrak{u}_{k}$ and is therefore dense in $\mathcal{U}$. For $f$ in the domain of $\Lambda_{Q}^{r}$ define the norm

$$
\|f\|_{Q, r}=\left\|\Lambda_{Q}^{r} f\right\|_{\mathcal{U}}
$$

The operator $\Lambda_{Q}^{r}$ extends to every tensor product $X \otimes \mathcal{U}^{\otimes n}$; we will keep the same notation for this extension and, in the case $X=\mathbb{R}$, for the corresponding norm (3.1). Denote by $(\mathcal{L})^{Q, r}(\mathbb{F})$ the dual space of $(\mathcal{L})_{Q, r}(\mathbb{F})$ relative to the inner product in $L_{2}(\mathbb{F})$. If $\eta \in(\mathcal{L})_{Q, r}(\mathbb{F} ; X)$ and $\zeta \in(\mathcal{L})^{Q, r}(\mathbb{F})$, then the duality

$$
\langle\langle\eta, \zeta\rangle\rangle
$$

is defined and is an element of $X$.

Definition 3.2. The space $(\mathcal{L})_{Q, r}(\mathbb{F} ; X)$ is the closure of the set of random elements of the form

$$
\eta=\eta_{0}+\sum_{k=1}^{N} \frac{1}{k !} \boldsymbol{\delta}^{\hat{\otimes} k}\left(\eta_{k}\right), \quad N \geq 1
$$


where $\eta_{0} \in X, \eta_{k} \in X \otimes \mathcal{U}^{\hat{\otimes} k}$, and each $\eta_{k}$ in the domain of $\Lambda_{Q}^{r}$, with respect to the norm

$$
\|\eta\|_{Q, r ; X}^{2}=\left\|\eta_{0}\right\|_{X}^{2}+\sum_{k=1}^{N} \frac{1}{(k !)^{2}}\|\| \eta_{k}\left\|_{Q, r}\right\|_{X}^{2} .
$$

Theorem 3.3. A formal series $\eta=\sum_{\alpha \in \mathcal{J}} \eta_{\alpha} \xi_{\alpha}$ is an element of $(\mathcal{L})_{Q, r}(\mathbb{F} ; X)$ if and only if

$$
\sum_{\alpha \in \mathcal{J}} \frac{1}{|\alpha| !} q^{2 r \alpha}\left\|\eta_{\alpha}\right\|_{X}^{2}<\infty
$$

The left-hand side of (3.3), if finite, is equal to $\|\eta\|_{Q, r ; X}^{2}$.

Proof. By orthonormality of $\xi_{\alpha}$, is enough to consider $\eta=f \xi_{\alpha}, f \in X$. Then (2.18) implies

$$
\|\eta\|_{Q, r ; X}^{2}=\frac{1}{(|\alpha| !)^{2} \alpha !}\left\|E_{\alpha}\right\|_{Q, r}^{2}\|f\|_{X}^{2}=\frac{1}{|\alpha| !}\|f\|_{X}^{2},
$$

because the definition of $E_{\alpha}$ implies

$$
\left\|E_{\alpha}\right\|_{Q, r}^{2}=q^{2 r \alpha}|\alpha| ! \alpha !
$$

Corollary 3.4. A formal series $\zeta=\sum_{\alpha \in \mathcal{J}} \zeta_{\alpha} \xi_{\alpha}$ is an element of the dual space $(\mathcal{L})^{Q, r}(\mathbb{F})$ if and only if

$$
\sum_{\alpha \in \mathcal{J}} q^{-2 r \alpha}\left|\zeta_{\alpha}\right|^{2}<\infty
$$

In this case,

$$
\langle\langle\eta, \zeta\rangle\rangle=\sum_{\alpha \in \mathcal{J}} \eta_{\alpha} \zeta_{\alpha}
$$

For $h \in \mathcal{U}$ define the stochastic exponential

$$
\mathcal{E}_{h}=\exp \left(\dot{W}(h)-\frac{1}{2}\|h\|_{\mathcal{U}}^{2}\right) .
$$

Proposition 3.5. $\mathcal{E}_{h} \in(\mathcal{L})^{Q, r}(\mathbb{F})$ if and only of $\|h\|_{Q,-r}<1$.

Proof. We apply Corollary 3.4. If $h=\sum_{k \geq 1} h_{k} \mathfrak{u}_{k}, h_{k} \in \mathbb{R}$, then by direct computation using the generating function of the Hermite polynomials,

$$
\mathcal{E}_{h}=\sum_{\alpha \in \mathcal{J}} \frac{h^{\alpha}}{\sqrt{\alpha !}} \xi_{\alpha}, h^{\alpha}=\prod_{k \geq 1} h_{k}^{\alpha_{k}} .
$$

Then, by the multinomial expansion,

$$
\sum_{\alpha \in \mathcal{J}} \frac{|\alpha| !}{\alpha !} h^{2 \alpha} q^{-2 r \alpha}=\sum_{n \geq 0}\left(\sum_{k \geq 1} h_{k}^{2} q_{k}^{-r}\right)^{n}=\sum_{n \geq 0}\left(\|h\|_{Q,-r}^{2}\right)^{n}
$$

and the result follows. 
In applications to evolutions equations, the number $r$ in the solution space $(\mathcal{L})_{Q, r ; X}$ is usually negative, as we want to help the series in (3.3) to converge. As a result, the following definition is appropriate.

Definition 3.6. We say the the element $h$ of $\mathcal{U}$ is sufficiently small relative to the sequence $Q$ if there exists a positive number $r$ such that

$$
\|h\|_{r, Q}<1 \text {. }
$$

\section{The Evolution Equation and Main Result}

Definition 4.1. The triple $\left(V, H, V^{\prime}\right)$ of Hilbert spaces is called normal if and only if

(1) $V \hookrightarrow H \hookrightarrow V^{\prime}$ and both embeddings $V \hookrightarrow H$ and $H \hookrightarrow V^{\prime}$ are dense and continuous;

(2) The space $V^{\prime}$ is the dual of $V$ relative to the inner product in $H$;

(3) There exists a constant $C>0$ such that $\left|(f, v)_{H}\right| \leq C\|v\|_{V}\|f\|_{V^{\prime}}$ for all $v \in V$ and $f \in H$.

For example, the Sobolev spaces $\left(H_{2}^{\ell+\gamma}\left(\mathbb{R}^{d}\right), H_{2}^{\ell}\left(\mathbb{R}^{d}\right), H_{2}^{\ell-\gamma}\left(\mathbb{R}^{d}\right)\right), \gamma>0, \ell \in \mathbb{R}$, form a normal triple.

Denote by $\left\langle v^{\prime}, v\right\rangle, v^{\prime} \in V^{\prime}, v \in V$, the duality between $V$ and $V^{\prime}$ relative to the inner product in $H$. The properties of the normal triple imply that $\left|\left\langle v^{\prime}, v\right\rangle\right| \leq C\|v\|_{V}\left\|v^{\prime}\right\|_{V^{\prime}}$, and, if $v^{\prime} \in H$ and $v \in V$, then $\left\langle v^{\prime}, v\right\rangle=\left(v^{\prime}, v\right)_{H}$.

We will also use the following notation:

$$
\mathcal{V}(T)=L_{2}((0, T) ; V), \mathcal{H}(T)=L_{2}((0, T) ; H), \mathcal{V}^{\prime}(T)=L_{2}\left((0, T) ; V^{\prime}\right) .
$$

Given a normal triple $\left(V, H, V^{\prime}\right)$, let $\mathbf{A}(t): V \rightarrow V^{\prime}$ and $\mathbf{M}(t): V \rightarrow V^{\prime} \otimes \mathcal{U}$ be bounded linear operators for every $t \in[0, T]$.

Definition 4.2. The solution of the stochastic evolution equation

$$
u(t)=u^{0}+\int_{0}^{t}(\mathbf{A}(s) u(s)+f(s)+\boldsymbol{\delta}(\mathbf{M}(s) u(s))) d s, 0 \leq t \leq T,
$$

with $f \in \bigcup_{r}(\mathcal{L})_{Q, r}\left(\mathbb{F} ; \mathcal{V}^{\prime}(T)\right)$ and $u_{0} \in \bigcup_{r}(\mathcal{L})_{Q, r}(\mathbb{F} ; H)$, is an object with the following properties:

(1) There exists a weight sequence $Q^{\prime}$ and a real number $r^{\prime}$ such that

$$
u \in(\mathcal{L})_{Q^{\prime}, r^{\prime}}(\mathbb{F} ; \mathcal{V}(T))
$$

(2) For every $h \in \mathcal{U}$ that is sufficiently small relative to the sequence $Q^{\prime}$, the function

satisfies

$$
u_{h}(t)=\left\langle\left\langle u(t), \mathcal{E}_{h}\right\rangle\right\rangle
$$

$$
u_{h}(t)=u_{h}(0)+\int_{0}^{t}\left(\mathbf{A}(s) u_{h}(s)+f_{h}(s)+\left(\mathbf{M}(s) u_{h}(s), h\right)_{\mathcal{U}}\right) d s
$$

in $\mathcal{V}^{\prime}(T)$ 
Remark 4.3. The solution described by Definition 4.2 belongs to the class of "variational solutions", which is quite typical for partial differential equations (see [5, 7, 8, 10], etc.) Indeed, direct computations show that $\mathbf{D} \mathcal{E}_{h}=h \mathcal{E}_{h}$, and then (4.3) is the result of a (formal) application of the duality $\left\langle\left\langle u, \mathcal{E}_{h}\right\rangle\right\rangle$ to both sides of (4.2).

Fix an orthonormal basis $\mathfrak{U}=\left\{\mathfrak{u}_{k}, k \geq 1\right\}$ in $\mathcal{U}$. Then, for every $v \in V$, there exists a collection $v_{k} \in V^{\prime}, k \geq 1$, such that

$$
\mathbf{M} v=\sum_{k \geq 1} v_{k} \otimes \mathfrak{u}_{k}
$$

We therefore define the operators $\mathbf{M}_{k}: V \rightarrow V^{\prime}$ by setting $\mathbf{M}_{k} v=v_{k}$ and write

$$
\mathbf{M} v=\sum_{k \geq 1}\left(\mathbf{M}_{k} v\right) \otimes \mathfrak{u}_{k}
$$

Then

$$
\boldsymbol{\delta}(\mathbf{M}(s) u(s))=\sum_{k \geq 1} \mathbf{M}_{k}(s) u(s)(t) \diamond \xi_{k}, \xi_{k}=\dot{W}\left(\mathfrak{u}_{k}\right),
$$

and equation (4.2) becomes

$$
u(t)=u^{0}+\int_{0}^{t}\left(\mathbf{A}(s) u(s)+f(s)+\sum_{k \geq 1} \mathbf{M}_{k}(s) u(s) \diamond \dot{W}\left(\mathfrak{u}_{k}\right)\right) d s .
$$

If the noise $\dot{W}$ itself depends on time, this dependence is encoded in the operator $\mathbf{M}$. As a result, (4.2) includes many popular evolution equations as particular cases.

Example 4.4. (1) Let us see how the elementary Itô equation $u(t)=1+\int_{0}^{t} u(s) d w(s)$, where $w$ is a standard Brownian motion, is a particular case of (4.2). We take $V=H=V^{\prime}=\mathbb{R}, \mathcal{U}=L_{2}((0, T))$, so that $\dot{W}=\dot{w}(t)=\sum_{k>1} \mathfrak{u}_{k}(t) \xi_{k}$ is the Gaussian white noise in time. Next, put $\mathbf{A}=0, \mathbf{M}_{k}(t) u(t)=u(t) \mathfrak{u}_{k}(t)$. Then $\boldsymbol{\delta}\left(\mathbf{M}_{k}(t) u(t)\right)=$ $u(t) \diamond \sum_{k \geq 1} \mathfrak{u}_{k}(t) \xi_{k}=u(t) \diamond \dot{w}(t)$. The equivalence of $u(t)=1+\int_{0}^{t} \boldsymbol{\delta}(\mathbf{M}(s) u(s)) d s$ and $u(t)=1+\int_{0}^{t} u(s) d w(s)$ now follows from the equality $\int_{0}^{t} u(s) \diamond \dot{w}(s) d s=\int_{0}^{t} u(s) d w(s)$; see [2, Section 2.5].

(2) Equations with space-time white noise correspond to $\mathcal{U}=L_{2}((0, T)) \times L_{2}(G)$, $G \subseteq \mathbb{R}^{d}$, so that the basis in $\mathcal{U}$ is naturally indexed by a pair of indices $i, k: \mathfrak{u}_{i k}=$ $m_{i}(t) h_{k}(x)$. Thus, equation $d u(t, x)=u_{x x} d t+u d W(t, x)$ is a particular case of (4.2) with $\mathbf{M}_{i k}(t) u(t, x)=m_{i}(t) h_{k}(x) u(t, x)$.

(3) Equations with fractional noise are also covered by (4.2). For example, consider $u(t)=1+\int_{0}^{t} u(s) d W^{H}(s)$, where $W^{H}$ is fractional Brownian motion and $H>1 / 2$. With the appropriate interpretation of the stochastic integral, we have

$$
\int_{0}^{t} u(s) d w^{H}(s)=\int_{0}^{t} u(s) \diamond \dot{w}^{H}(s) d s
$$

where

$$
\dot{w}^{H}(t)=\sum_{k \geq 1} m_{k}^{H}(t) \xi_{k}
$$


and $m_{k}^{H}$ are suitable elements of $L_{2}((0, T)$ ) (see for example [9, Chapter 5]. Then, in (4.2), we take $\mathcal{U}=L_{2}((0, T))$ and $\mathbf{M}_{k}(t) u(t)=m_{k}^{H}(t) u(t)$.

Let us fix an orthonormal basis $\mathfrak{U}$ in $\mathcal{U}$ so that equation (4.2) becomes (4.5). With $\xi_{k}=\dot{W}\left(\mathfrak{u}_{k}\right)$, define $\xi_{\alpha}, \alpha \in \mathcal{J}$ according to (2.2). Given a Hilbert space $X$, every element $\eta$ of $(\mathcal{L})_{Q, r}(\mathbb{F} ; X)$ can be written as a formal series $\sum_{\alpha} \eta_{\alpha} \xi_{\alpha}$ with $\eta_{\alpha} \in X$ satisfying (3.3). The following theorem provides a necessary and sufficient condition for the formal series $\sum_{\alpha} u_{\alpha}(t) \xi_{\alpha}$ to be a solution of (4.5).

Theorem 4.5. Let $u=\sum_{\alpha \in \mathcal{J}} u_{\alpha} \xi_{\alpha}$ be an element of $(\mathcal{L})_{Q^{\prime}, r^{\prime}}(\mathbb{F} ; \mathcal{V}(T))$. The process $u$ is a solution of equation (4.5) if and only if the functions $u_{\alpha}$ have the following properties:

(1) every $u_{\alpha}$ is an element of $\left.\mathbf{C}([0, T] ; H)\right)$.

(2) the system of equalities

$$
u_{\alpha}(t)=u_{\alpha}^{0}+\int_{0}^{t}\left(\mathbf{A}(s) u_{\alpha}(s)+f_{\alpha}(s)+\sum_{k \geq 1} \sqrt{\alpha_{k}} \mathbf{M}_{k}(s) u_{\alpha-\varepsilon_{k}}(s)\right) d s
$$

holds in $\mathcal{V}^{\prime}(T)$ for all $\alpha \in \mathcal{J}$.

Proof. If $h=\sum_{k} h_{k} \mathfrak{u}_{k}$, then, by (3.5) and Corollary $3.4 u_{h}=\sum_{\alpha \in \mathcal{J}} \frac{u_{\alpha} h^{\alpha}}{\sqrt{\alpha !}}$. With no loss of generality we can assume that $\sum_{k \geq 1}\left(q_{k}^{\prime}\right)^{2}<\infty$. Then, by a general result from functional analysis [4], the mapping $u_{h}: \mathcal{U} \mapsto \mathcal{V}(T)$ is analytic at zero. By direct computation,

(1) if $u_{h}$ satisfies (4.3), then

$$
u_{\alpha}=\left.\frac{1}{\sqrt{\alpha !}} \frac{\partial^{|\alpha|} u_{h}}{\partial h_{1}^{\alpha_{1}} h_{2}^{\alpha_{2}} \ldots}\right|_{h=0}
$$

and each $u_{\alpha}$ satisfies (4.6).

(2) if $u_{\alpha}$ satisfies (4.6) and $u_{h}=\sum_{\alpha} u_{\alpha} h^{\alpha}$, then $u_{h}$ satisfies (4.3).

This simple but very helpful result establishes the equivalence of the "physical" (4.5) and the (stochastic) Fourier (4.6) forms of equation (4.2). The system of equations (4.6) is often referred in the literature as the propagator of equation (4.5). Note that the propagator is lower-triangular and can be solved by induction on $|\alpha|$.

To prove existence and uniqueness of solution of (4.2), we make the following assumptions about the operators $\mathbf{A}$ and $\mathbf{M}$.

(A): For every $U_{0} \in H$ and $F \in \mathcal{V}^{\prime}(T)$, there exists a unique function $U \in \mathcal{V}$ that solves the deterministic equation

$$
U(t)=U_{0}+\mathbf{A}(t) U(t)+F(t),
$$

and there exists a constant $C=C(\mathbf{A}, T)$ so that

$$
\|U\|_{\mathcal{V}(T)} \leq C(\mathbf{A}, T)\left(\left\|U_{0}\right\|_{H}+\|F\|_{\mathcal{V}^{\prime}(T)}\right) .
$$


In particular, the operator $\mathbf{A}$ generates a semi-group $\Phi=\Phi_{t, s}, t \geq s \geq 0$, and

$$
U(t)=\Phi_{t, 0} U_{0}+\int_{0}^{t} \Phi_{t, s} F(s) d s .
$$

$(\mathrm{M})$ : For every $v \in \mathcal{V}(T)$ and $k \geq 1$,

$$
\int_{0}^{T}\left\|\int_{0}^{t} \Phi_{t, s} \mathbf{M}_{k}(s) v(s) d s\right\|_{V}^{2} d t \leq C_{k}^{2}\|v\|_{\mathcal{V}(T)}^{2}
$$

with numbers $C_{k}$ independent of $v$.

Remark 4.6. There are various types of assumptions on the operator $\mathbf{A}$ that yield the statement of the assumption (A). In particular, (A) holds if the operator $\mathbf{A}$ is coercive in $\left(V, H, V^{\prime}\right)$ :

$$
\langle\mathbf{A}(t) v, v\rangle+\gamma\|v\|_{V}^{2} \leq C\|v\|_{H}^{2}
$$

for every $v \in V, t \in[0, T]$, where $\gamma>0$ and $C \in \mathbb{R}$ are both independent of $v, t$.

The following theorem is the main result of this paper.

Theorem 4.7. In addition to $(A)$ and $(M)$, assume that, for some positive number $r$ and $a$ weight sequence $Q, u^{0} \in(\mathcal{L})_{Q,-r}(\mathbb{F} ; H)$ and $f \in(\mathcal{L})_{Q,-r}\left(\mathbb{W} ; \mathcal{V}^{\prime}(T)\right)$. If

$$
\sum_{\alpha \in \mathcal{J}} \frac{1}{q^{2 r \alpha} \alpha !}<\infty
$$

then there exists a weight sequence $Q^{\circ}$ and a negative real number $r^{\circ}$ such that equation (4.2) has a unique solution $u \in(\mathfrak{L})_{Q^{\circ}, r^{\circ}}(\mathbb{F} ; \mathcal{V}(T))$ and

$$
\|u\|_{Q^{\circ}, r^{\circ} ; \mathcal{V}(T)}^{2} \leq C \cdot\left(\left\|u^{0}\right\|_{Q, r ; H}^{2}+\|f\|_{Q, r ; \mathcal{V}^{\prime}(T)}^{2}\right) .
$$

The number $C>0$ depends only on $Q, r, T$ and the operators $\mathbf{A}, \mathbf{M}$.

Proof. The proof consists of two steps: first, we prove the result for deterministic functions $u^{0}, f$ and then use linearity to extend the result to the general case.

Step 1. Assume that the functions $u^{0} \in H, f \in \mathcal{V}^{\prime}(T)$ are deterministic so that $u_{h}^{0}=u^{0}, f_{h}=f$. By assumptions (4.8) and (4.9) the evolution equation

$$
u_{h}(t)=u^{0}+\int_{0}^{t}\left(\mathbf{A}(s) u_{h}(s)+f(s)+\sum_{k=1}^{\infty} \mathbf{M}_{k}(s) u_{h}(s) h_{k}\right)
$$

has a unique solution in $\mathcal{V}(T)$ as long as $\sum_{k \geq 1} h_{k}^{2}$ is small enough; since the equation is linear, the solution is an analytic function of $h_{k}$.

Next,

$$
u(t)=\sum_{\alpha \in \mathcal{J}} u_{\alpha}(t) \xi_{\alpha}
$$


and, by (4.6), the coefficients $u_{\alpha}$ satisfy

$$
\begin{aligned}
& u_{(0)}(t)=u^{0}+\int_{0}^{t}\left(\mathbf{A}(s) u_{(0)}(s)+f(s)\right) d s, \quad|\alpha|=0 \\
& u_{\epsilon_{k}}(t)=\int_{0}^{t} \mathbf{A}(s) u_{\epsilon_{k}}(s) d s+\int_{0}^{t} \mathbf{M}_{k}(s) u_{(0)}(s) d s, \quad|\alpha|=1 ; \\
& u_{\alpha}(s)=\int_{0}^{t} \mathbf{A}(s) u_{\alpha}(s) d s+\sum_{k} \sqrt{\alpha_{k}} \int_{0}^{t} \mathbf{M}_{k}(s) u_{\alpha-\epsilon_{k}}(s) d s,|\alpha|>1 .
\end{aligned}
$$

Denote by $\Phi=\Phi_{s, t}, t \geq s \geq 0$ the semigroup generated by the operator $\mathbf{A}(t)$. It follows by induction on $|\alpha|$ that

$$
\begin{aligned}
u_{(0)}(t) & =\Phi_{t, 0} u^{0}(x)+\int_{0}^{t} \Phi_{t, s} f(s) d s,|\alpha|=0 \\
u_{\epsilon_{k}}(t) & =\int_{0}^{t} \Phi_{t, s} \mathbf{M}_{k}(s) u_{(0)}(s) d s,|\alpha|=1 \\
u_{\alpha}(t) & =\frac{1}{\sqrt{\alpha !}} \int_{0}^{t} \int_{0}^{s_{n}} \cdots \int_{0}^{s_{2}} \Phi_{t, s_{n}} \mathbf{M}_{i_{n}} \Phi_{s_{n}, s_{n-1}} \cdots \mathbf{M}_{i_{n-1}} \Phi_{s_{2}, s_{1}} \mathbf{M}_{i_{1}} u_{(0)} d s_{1} \ldots d s_{n} \\
& |\alpha|=n>1, K_{\alpha}=\left\{i_{1}, \ldots, i_{n}\right\} .
\end{aligned}
$$

By assumptions (4.8) and (4.9),

$$
\left\|u_{\alpha}(t)\right\|_{\mathcal{V}(T)}^{2} \leq \frac{(|\alpha| !)^{2}}{\alpha !}\left(\left\|u^{0}\right\|_{H}^{2}+\|f\|_{\mathcal{V}^{\prime}(T)}^{2}\right) \prod_{k \geq 1} C_{k}^{2 \alpha_{k}}
$$

It is known (see, for example, [2, pages 31,35$]$ ) that

$$
|\alpha| ! \leq \alpha !(2 \mathbb{N})^{2 \alpha}, \quad \sum_{\alpha \in \mathcal{J}}(2 \mathbb{N})^{-r \alpha}<\infty \text { if and only if } r>1
$$

where

$$
(2 \mathbb{N})^{2 \alpha}=2^{2|\alpha|} \prod_{k \geq 1} k^{2 \alpha_{k}}
$$

Define the sequence $Q^{\circ}=\left\{q_{k}^{\circ}, k \geq 1\right\}$ by

$$
q_{k}^{\circ}=2 k\left(1+C_{k}\right) \text {. }
$$

Then (3.3) and (4.15) imply

$$
\|u\|_{Q^{\circ}, r^{\circ} ; \mathcal{V}(T)}^{2} \leq C \cdot\left(\left\|u^{0}\right\|_{H}^{2}+\|f(t)\|_{\mathcal{V}^{\prime}(T)}^{2}\right) \text { for every } r^{\circ}<-2 .
$$

Step 2. As in Step 1, existence and uniqueness of solution follows from unique solvability of the parabolic equation (4.11), and it remains to establish (4.10).

Given $v \in H, F \in \mathcal{V}^{\prime}(T)$, and $\gamma \in \mathcal{J}$, denote by $u(t ; v, F, \gamma)$ the solution of (4.2) with $u^{0}=v \xi_{\gamma}, f=F \xi_{\gamma}$. If $u^{0}=\sum_{\alpha \in \mathcal{J}} u_{\alpha}^{0} \xi_{\alpha}, f=\sum_{\alpha \in \mathcal{J}} f_{\alpha} \xi_{\alpha}$, then, by linearity,

$$
u(t)=\sum_{\gamma \in \mathcal{J}} u\left(t ; u_{\gamma}^{0}, f_{\gamma}, \gamma\right)
$$


It follows from (4.13) that $u_{\alpha}(t ; v, F, \gamma)=0$ if $|\alpha|<|\gamma|$ and

$$
\frac{u_{\alpha+\gamma}(t ; v, F, \gamma)}{\sqrt{(\alpha+\gamma) !}}=\frac{u_{\alpha}\left(t ; \frac{v}{\sqrt{\gamma !}}, \frac{F}{\sqrt{\gamma !}},(0)\right)}{\sqrt{\alpha !}} .
$$

Using the results of Step 1,

$$
\left\|u\left(t ; u_{\gamma}^{0}, f_{\gamma}, \gamma\right)\right\|_{Q^{\circ}, r^{\circ} ; \mathcal{V}(T)} \leq \frac{C}{\sqrt{\gamma !}}\left(\left\|v_{\gamma}\right\|_{H}+\left\|f_{\gamma}(t)\right\|_{\mathcal{V}^{\prime}(T)}\right) .
$$

With $Q^{\circ}$ defined in (4.17) and $r^{\circ}<-2$, (4.10) now follows from (4.18) by the triangle and the Cauchy-Schwartz inequalities.

Corollary 4.8 (A generalization of the Krylov-Veretennikov formula from [6]). Assume that $u^{0}$ and $f$ are deterministic and take $q_{k}^{\circ}$ from (4.17). Define the sequence $U_{n}=U_{n}(t), n \geq 0$ by induction

$$
U_{0}(t)=u_{(0)}(t), U_{n+1}(t)=\int_{0}^{t} \Phi_{t, s} \boldsymbol{\delta}\left(\mathbf{M}^{\circ}(s) U_{n}(s)\right) d s, n>0,
$$

where $\mathbf{M}^{\circ}=\left(q_{1}^{\circ} \mathbf{M}_{1}, q_{2}^{\circ} \mathbf{M}_{2}, \ldots\right)$. Then

$$
\sum_{|\alpha|=n}\left(q^{\circ}\right)^{\alpha} u_{\alpha}(t) \xi_{\alpha}=U_{n}(t)
$$

Proof. By (4.13),

$$
\left(q^{\circ}\right)^{\alpha} u_{\alpha}(t)=\int_{0}^{t} \mathbf{A}\left(q^{\circ}\right)^{\alpha} u_{\alpha}(s) d s+\sum_{k \geq 1} \int_{0}^{t} q_{k} \sqrt{\alpha_{k}} \mathbf{M}_{k}\left(q^{\circ}\right)^{\alpha-\varepsilon_{k}} u_{\alpha-\varepsilon_{k}}(s) d s .
$$

Therefore,

$$
\left(U_{n}(t)\right)_{\alpha}=\sum_{k \geq 1} \sqrt{\alpha_{k}} \int_{0}^{t} \Phi_{t-s} \mathbf{M}_{k}^{\circ}\left(U_{n-1}(s)\right)_{\alpha-\varepsilon_{k}} d s
$$

By (2.13),

$$
U_{n}(t)=\int_{0}^{t} \Phi_{t, s} \boldsymbol{\delta}\left(\mathbf{M}^{\circ}(s) U_{n-1}(s)\right) d s
$$

and the result follows.

Remark 4.9. The main goal of Theorem 4.7 is universality: to cover the largest possible class of equations. It is therefore inevitable, and natural, that, for many particular equations, there are much better regularity results than (4.10).

\section{REFERENCES}

[1] R. H. Cameron and W. T. Martin, The orthogonal development of nonlinear functionals in a series of Fourier-Hermite functions, Ann. Math. 48 (1947), no. 2, 385-392.

[2] H. Holden, B. Øksendal, J. Ubøe, and T. Zhang, Stochastic partial differential equations, Birkhäuser, Boston, 1996.

[3] K. Ito, Multiple Wiener integral, J. Math. Soc. Japan 3 (1951), 157-169.

[4] Yu. G. Kondratiev, P. Leukert, J. Potthoff, L. Streit, and W. Westerkamp, Generalized functionals in Gaussian spaces: the characterization theorem revisited, J. Funct. Anal. 141 (1996), no. 2, 301-318. 
[5] N. V. Krylov, Introduction to the theory of diffusion processes, American Mathematical Society, Providence, RI, 1995.

[6] N. V. Krylov and A. J. Veretennikov, On explicit formula for solutions of stochastic equations, Math. USSR Sbornik 29 (1976), no. 2, 239-256.

[7] J.-L. Lions, Quelques méthodes de résolution des problèmes aux limites non linéaires, Dunod, Paris, 1969.

[8] J.-L. Lions and E. Magenes, Problémes aux limites non homogènes et applications, volume 1, Dunod, Paris, 1968.

[9] D. Nualart, Malliavin calculus and related topics, 2nd edition, Springer, New York, 2006.

[10] B. L. Rozovskii, Stochastic evolution systems, Kluwer Academic Publishers, Dordrecht, 1990.

Current address, S. V. Lototsky: Department of Mathematics, USC, Los Angeles, CA 90089

E-mail address, S. V. Lototsky: lototsky@math.usc.edu

$U R L:$ http://math.usc.edu/ lototsky

Current address, B. L. Rozovskii: Division of Applied Mathematics, Brown University, Providence, RI 02912

E-mail address, B. L. Rozovskii: rozovsky@dam.brown.edu

URL: http://www.dam.brown.edu/people/rozovsky.html 